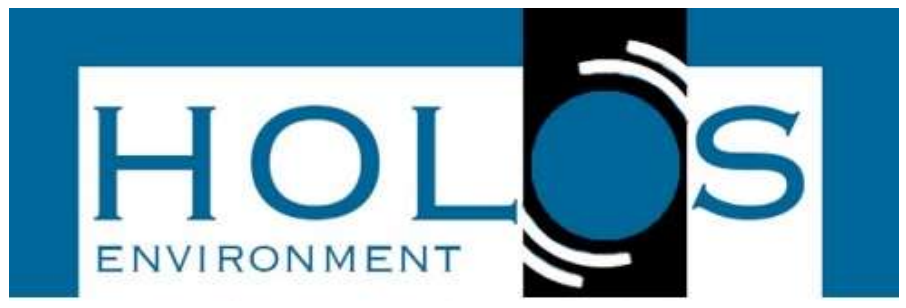

\title{
INTRUSÃO DE VAPORES DO SOLO: BREVE HISTÓRICO SOBRE DESENVOLVIMENTO DA TECNOLOGIA, CENÁRIO BRASILEIRO E AVANÇOS RECENTES
}

\section{SOIL VAPOR INTRUSION: BRIEF HISTORY ON TECHNOLOGY DEVELOPMENT, BRAZILIAN SCENARIO AND RECENT ADVANCES}

\author{
José Carlos Rocha Gouvêa Júnior ${ }^{1}$; Reginaldo Antônio Bertolo1; \\ Sasha Tom Hart ${ }^{1}$
}

Artigo recebido em: 16/08/2018 e aceito para publicação em: 14/11/2018.

DOI: http://dx.doi.org/10.14295/holos.v18i2.12307

Resumo: A intrusão de vapores do solo, processo definido pela migração de contaminantes em fase volátil desde uma fonte de contaminação existente em subsuperfície para o interior de edificações, tem sido tema de debates entre comunidades científicas e regulatórias internacionais há aproximadamente três décadas. No Brasil, como é o caso para a maioria das tecnologias em desenvolvimento, as instruções técnicas para avaliar a intrusão de vapores provenientes da América do Norte e da Europa vêm sendo adaptadas de forma a possibilitar a sua aplicação a nível local. A nível mundial, verifica-se que a abordagem para avaliação da intrusão de vapores tem tipicamente sido focada na utilização de modelos preditivos e na avaliação de riscos à saúde humana (ARSH), sendo que investigações de campo e a utilização de múltiplas linhas de evidência fazem parte da regulamentação apenas em poucos países (Austrália, Canadá, Dinamarca, Estados Unidos e Reino Unido). Nestes locais, novas tecnologias e formas de abordagem vêm sendo utilizadas de maneira bem-sucedida para elucidar os cenários de exposição, por meio do refinamento de modelos conceituais, e assim auxiliar a tomada de decisões. No Brasil, foram identificados desenvolvimentos com relação ao quadro regulatório e tecnológico, porém existe um campo amplo de oportunidades para o estabelecimento de diretrizes e procedimentos específicos para avaliação do processo de intrusão de vapores.

Palavras-chave: Intrusão de Vapores. Quadro regulatório. Modelo Conceitual. Múltiplas Linhas de Evidência. Avanços Tecnológicos.

\begin{abstract}
Soil vapor intrusion is a process defined as volatile compounds migration from a subsurface source to indoor air, and it has been subject of debate among international regulatory and scientific communities for nearly three decades. In Brazil, as is the case with most developing technologies, technical guidelines for the assessment of soil vapor intrusion have been adapted, from the ones developed in North America and in Europe. Globally, the usual approach for the assessment of soil vapor intrusion is typically focused on the use of predictive modeling and human health risk assessment, while field investigations and use of multiple lines of evidence are regulated only in a few countries (Australia, Canada, Denmark, United States and United Kingdom). In these countries, innovative approach and new technologies have been successfully applied in order to explain the vapor intrusion process, refine conceptual models and support the decision-making process. In Brazil, advances on technological and regulatory framework have been identified, but there is also a wide scope of opportunities for technological advances and the establishment of specific guidelines for evaluation of the soil vapor intrusion process.
\end{abstract}

\footnotetext{
${ }^{1}$ Instituto de Geociências da Universidade de São Paulo - Centro de Pesquisas de Águas Subterrâneas. E-mails: (jcrgouveaj@outlook.com, bertolo@usp.br, sasha@elementarambiental.com.br)
} 
Palavras-chave: Soil Vapor Intrusion. Regulatory Framework. Conceptual Model. Multiple Lines of Evidence. Technological Advances.

\section{INTRODUÇÃO}

O processo de intrusão de vapores (Figura 01), pode ser definido como a migração de contaminantes em fase volátil desde uma fonte subsuperficial, presente no solo, águas subterrâneas ou ambos, para o interior de edificações e outras estruturas presentes na superfície (US EPA, 2002).

Figura 01 - Base conceitual do processo de migração de vapores subsuperficiais para ambientes fechados

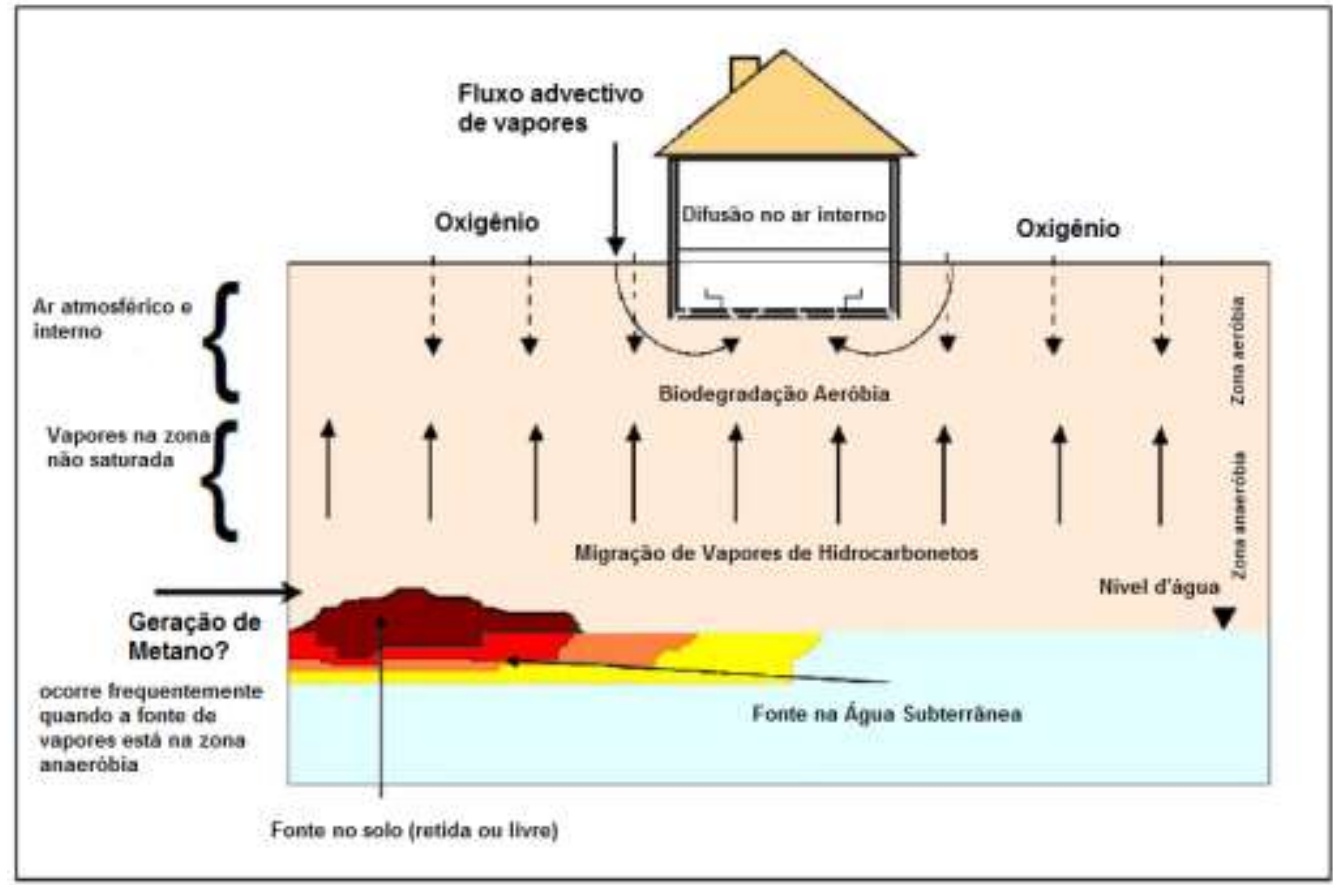

Fonte: Modificado de Api (2005)

Em casos extremos, os vapores acumulados podem representar riscos iminentes, como por exemplo, o de explosão ou tóxicos. Entretanto, na maior parte dos casos, são observadas apenas baixas concentrações, que, em um longo tempo de exposição, podem incrementar o risco da ocorrência de efeitos crônicos à saúde humana (US EPA, 2002).

A partir do fim da década de 90, o tema passou a receber maior atenção por parte das agências ambientais, principalmente nos Estados Unidos e na Europa, onde foram divulgados guias para orientar a condução de investigações focadas na avaliação da intrusão de vapores (MCHUGH e NICKELS, 2008). 
No Brasil, a partir do início dos anos 2000, como por exemplo na adoção da metodologia Ações Corretivas Baseadas em Risco - ACBR (CETESB, 2001), verificou-se que diversas áreas apresentavam situações potenciais de risco relacionadas a intrusão de vapores. Estas situações foram verificadas por meio da aplicação de modelos matemáticos e durante Avaliações de Riscos à Saúde Humana (ARSH), que utilizavam dados obtidos em amostras de solos e águas subterrâneas para prever concentrações de Compostos Orgânicos Voláteis (COVs) presentes no ar de ambientes fechados.

Este tipo de abordagem, muitas vezes apontada como conservadora, somada à ausência de diretrizes e padrões de comparação específicos, gerou uma crescente preocupação por parte dos órgãos governamentais brasileiros e dos demais stakeholders envolvidos (CHIARANDA, 2006; GOUVÊA, 2011). Como resultado, o processo de intrusão de vapores passou a ser mais intensamente estudado e, como é o caso para a maioria das tecnologias em desenvolvimento, as instruções técnicas para realizar este tipo de avaliação provenientes da América do Norte e da Europa passaram a ser adaptadas à realidade das condições brasileiras (NICOLE BRASIL, 2016).

Neste contexto, este artigo tem como objetivos apresentar i) uma revisão sobre o estado de desenvolvimento da tecnologia e dos avanços verificados no quadro regulatório a nível global e brasileiro; ii) um panorama sobre as técnicas recentes desenvolvidas internacionalmente para elaboração de modelos conceituais onde o processo de intrusão de vapores é relevante e, iii) o uso de abordagens inovadoras para determinar a ocorrência do processo de intrusão de vapores.

\section{DESENVOLVIMENTO DA TECNOLOGIA}

O reconhecimento do processo de migração de vapores subsuperficiais para ambientes fechados tem sido tema de debate entre as comunidades científicas e regulatórias há mais de três décadas. Inicialmente, o foco das pesquisas estava limitado ao mapeamento da distribuição de contaminantes, migração de radônio e de gases provenientes de aterros sanitários para ambientes fechados (MCHUGH; NICKELS, 2008).

No início dos anos 90, o processo de intrusão de vapores atraiu o interesse das agências ambientais norte americanas durante a implementação dos conceitos definidos por meio das ações corretivas baseadas em risco a saúde humana (Risk Based 
Corrective Action) e a partir da publicação do modelo de Johnson e Ettinger (JOHNSON; ETTINGER 1991).

O modelo de Johnson e Ettinger (J\&E), implementou algumas funções originalmente utilizadas nos modelos de intrusão de radônio para representar os fluxos difusivo e advectivo a partir de uma fonte subsuperficial. Os resultados deste modelo fornecem um fator de atenuação $(\alpha)$, que considera a profundidade da fonte de vapores e as características geotécnicas específicas das áreas avaliadas. A partir da utilização dos fatores de atenuação específicos, são estimadas as concentrações de vapores esperadas para o ar interno, possibilitando uma avaliação da exposição a estas concentrações em termos de risco à saúde humana. A Figura 02 a seguir demonstra a variação esperada para os fatores de atenuação em função da profundidade e do tipo de solo (USEPA 2002).

Figura 02- Fator de atenuação para vapores no solo em função da profundidade da fonte de vapores em fase dissolvida

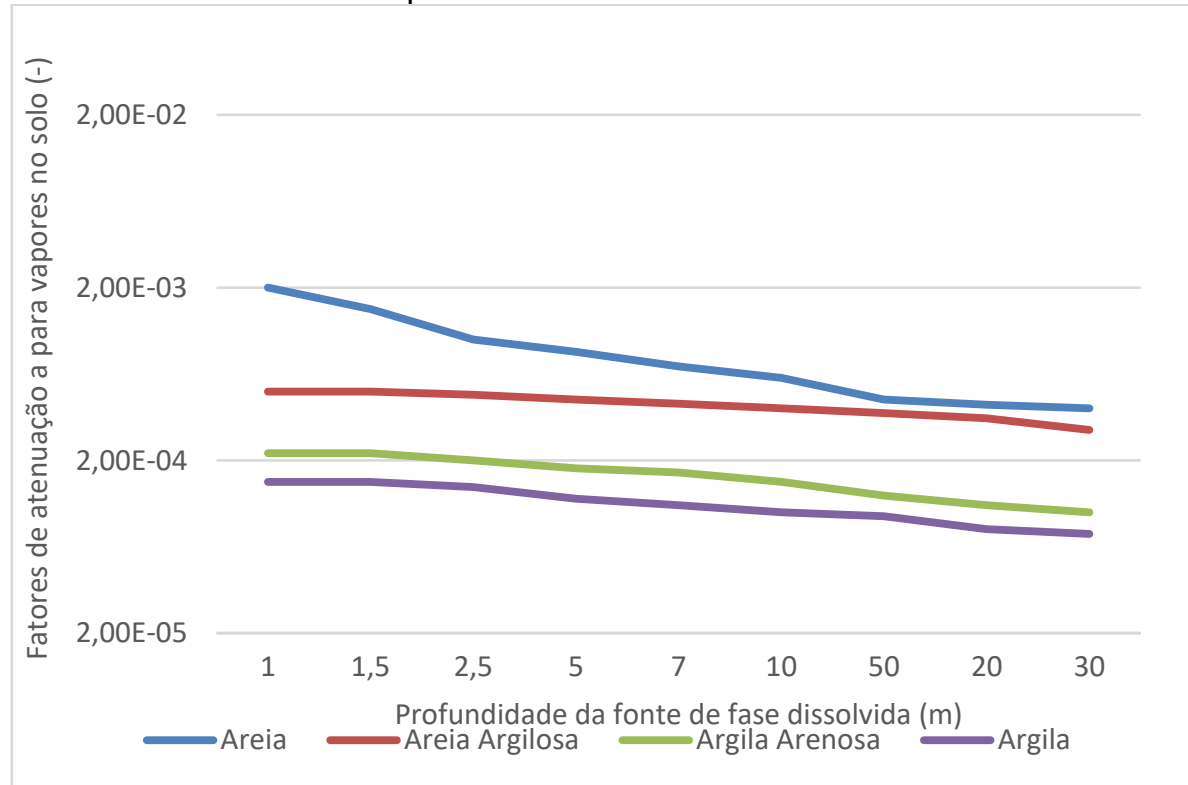

Fonte: Modificado de (USEPA, 2002)

Na segunda metade da década de 1990, a US EPA (US EPA, 2002) utilizou o modelo de J\&E, associado a algumas premissas conservadoras para calcular níveis alvos baseados em risco, de forma a prever o grau de exposição dos receptores ao ar interno impactado pela presença de COVs provenientes de fontes subsuperficiais.

A partir de 2002, a US EPA passou a limitar a utilização de modelagens, recomendando a utilização de dados obtidos em campo. Apesar disso, a US EPA procura incentivar a utilização do maior número de linhas de evidências, para verificar se a 
intrusão de vapores realmente ocorre na área avaliada. Embora a US EPA (US EPA, 2002), tenha limitado o uso de modelos preditivos, o modelo de J\&E ainda é amplamente utilizado (MCHUGH e NICKELS, 2008).

A partir da revisão de diversos guias existentes a nível mundial, verifica-se que a abordagem mais comumente utilizada é focada no uso de modelos numéricos e de Análise de Risco a Saúde Humana (ARSH). Investigações de campo possuem procedimentos estabelecidos apenas na Austrália, Canadá, Dinamarca, Estados Unidos e Reino Unido (MCHUGH et al, 2017).

A Tabela 01 a seguir, apresenta uma comparação entre os aspectos e diretrizes previstas nos guias da Austrália, Canadá, Dinamarca, Estados Unidos e Reino Unido.

Tabela 01 - Comparação entre aspectos e diretrizes previstas nos guias sobre o processo de avaliação de intrusão de vapores subsuperficiais da Austrália, Canadá, Dinamarca, Estados Unidos e Reino Unido

\begin{tabular}{|c|c|c|c|c|c|}
\hline \multirow{2}{*}{ Aspecto/Procedimento abordado } & \multicolumn{5}{|c|}{$\begin{array}{l}\text { Guias para avaliação da intrusão de vapores do } \\
\text { solo }\end{array}$} \\
\hline & Austrália & Canadá & Dinamarca & $\begin{array}{l}\text { Estados } \\
\text { Unidos }\end{array}$ & $\begin{array}{l}\text { Reino } \\
\text { Unido }\end{array}$ \\
\hline Revisão Conceitual sobre o processo de Intrusão de Vapores do Solo & $\checkmark$ & $\checkmark$ & $\checkmark$ & $\checkmark$ & $\checkmark$ \\
\hline Uso de Matrizes de Decisão & $\checkmark$ & $\checkmark$ & $\mathbf{X}$ & $\checkmark$ & $\mathbf{X}$ \\
\hline $\begin{array}{l}\text { Uso de Critérios Qualitativos (exclusão, priorização, necessidade de } \\
\text { avaliação/ detalhamento) }\end{array}$ & $\mathbf{X}$ & $\checkmark$ & $\mathbf{X}$ & $\checkmark$ & $\checkmark$ \\
\hline Procedimento para Adoção de Medidas Emergenciais & $\mathbf{X}$ & $\checkmark$ & $\checkmark$ & $\checkmark$ & $\mathbf{X}$ \\
\hline Procedimentos de Campo para Investigação e Coleta de Amostras & $\checkmark$ & $\checkmark$ & $\checkmark$ & $\checkmark$ & $\checkmark$ \\
\hline Avaliação de Hidrocarbonetos & $\checkmark$ & $\checkmark$ & $\checkmark$ & $\checkmark$ & $\checkmark$ \\
\hline Avaliação de Compostos Organoclorados & $\mathbf{X}$ & $\checkmark$ & $\checkmark$ & $\checkmark$ & $\mathbf{X}$ \\
\hline $\begin{array}{l}\text { Avaliação de Gases (Ex.: Aterros Sanitários, Radônio, Rede de Es- } \\
\text { gotos) }\end{array}$ & $\mathbf{x}$ & $\mathbf{X}$ & $\checkmark$ & $\checkmark$ & $\mathbf{x}$ \\
\hline Inclusão de Múltiplas Linhas de Evidência & $\mathbf{X}$ & $\mathbf{X}$ & $\checkmark$ & $\checkmark$ & $\mathbf{X}$ \\
\hline $\begin{array}{l}\text { Identificação de Pontos de Ingresso dos Vapores Subsuperficiais } \\
\text { para o Interior das Construções }\end{array}$ & $\mathbf{X}$ & $\mathbf{x}$ & $\checkmark$ & $\checkmark$ & $\mathbf{X}$ \\
\hline Uso de Valores de Referência Semi-Específicos & $\checkmark$ & $\checkmark$ & $\mathbf{X}$ & $\checkmark$ & $\checkmark$ \\
\hline Utilização de Modelos Numéricos & $\checkmark$ & $\checkmark$ & $\checkmark$ & $\checkmark$ & $\checkmark$ \\
\hline $\begin{array}{l}\text { Método para Cálculo de Valores de Referência Específicos para o } \\
\text { Site }\end{array}$ & X & $\checkmark$ & $\checkmark$ & $\checkmark$ & $\checkmark$ \\
\hline $\begin{array}{l}\text { Múltiplas Campanhas de Amostragens para Caracterização da Vari- } \\
\text { abilidade Temporal }\end{array}$ & $\mathbf{X}$ & $\mathbf{X}$ & $\mathbf{X}$ & $\checkmark$ & $\mathbf{X}$ \\
\hline Procedimentos para Elaboração de Modelos Conceituais & $\checkmark$ & $\checkmark$ & $\mathbf{X}$ & $\checkmark$ & $\checkmark$ \\
\hline Procedimentos para Interpretação de Dados & $\checkmark$ & $\checkmark$ & $\mathbf{X}$ & $\checkmark$ & $\checkmark$ \\
\hline Procedimentos para Mitigação de Riscos & $\mathbf{X}$ & $\mathbf{X}$ & $\mathbf{X}$ & $\checkmark$ & $\mathbf{X}$ \\
\hline Procedimentos para Monitoramento & $\mathbf{X}$ & $\mathbf{X}$ & $\mathbf{X}$ & $\checkmark$ & $\mathbf{X}$ \\
\hline Comunicação de Riscos & $\mathbf{X}$ & $X$ & $\mathbf{X}$ & $\checkmark$ & $X$ \\
\hline
\end{tabular}

$\checkmark \quad$ : Aspecto ou diretriz prevista no guia $\quad$ X: Aspecto ou diretriz não contemplada no guia.

As publicações de referência nestes países, quanto ao estabelecimento diretrizes para avaliação da intrusão de vapores, são descritas a seguir: 
Austrália: O guia australiano é focado na avaliação de áreas contaminadas pela presença de hidrocarbonetos de petróleo. O guia apresenta uma série de matrizes de decisão, que incorporam fluxogramas e detalhes operacionais, desde a seleção de procedimentos de amostragem até a interpretação de dados. Apesar de incluir diversos aspectos conceituais genéricos aplicáveis a uma série de COVs, as matrizes de decisão incluídas neste guia não são aplicáveis a COVs clorados e gases provenientes de aterros sanitários (CRCCARE, 2013).

Canadá: O guia federal canadense (HEALTH CANADA, 2010) inclui, em sua abordagem, duas fases de avaliação. Na primeira etapa, é realizada uma avaliação qualitativa para categorizar a área de acordo com seu potencial para ocorrência de intrusão de vapores. Esta avaliação irá determinar a necessidade de realização de uma segunda fase, que consiste em uma avaliação de riscos quantitativa, onde são utilizados fatores de atenuação de vapores semi-específicos para estimar as concentrações esperadas para o ar interno e assim prever potenciais riscos à saúde humana. Adicionalmente, o guia apresenta uma metodologia para cálculo de níveis alvo para vapores e águas subterrâneas, baseada no modelo de J\&E.

Dinamarca: O guia dinamarquês, publicado em 2003, inclui uma descrição de diversas técnicas disponíveis para a investigação da intrusão de vapores e a aplicação potencial destes métodos em função das características específicas das áreas. Os métodos apresentados são aplicados a nível de escala de edificações individuais, onde o foco é voltado para a identificação de pontos de ingresso dos vapores subsuperficiais para o interior das construções (DANISH EPA, 2003).

Estados Unidos: Nos Estados Unidos, os procedimentos são estabelecidos a nível federal pelo Technical Guide for Addressing Petroleum Vapor Intrusion at Leaking Underground Storage Tank Sites, de 2015 (US EPA, 2015). O guia americano recomenda a realização de múltiplas campanhas de amostragens para caracterização da variabilidade temporal, além da inclusão de múltiplas linhas de evidência para avaliação dos resultados obtidos ao longo dos processos de investigação. Adicionalmente, inclui diretrizes para comunicação de riscos e adoção de medidas de mitigação (US EPA, 2015). Atualmente, diversos estados norte-americanos possuem métodos 
próprios para avaliação da intrusão de vapores, com abordagem similar a recomendada pela USEPA (NEW JERSEY, 2005).

Reino Unido: No Reino Unido, são seguidas as recomendações descritas no relatório técnico Vapour Transfer of Soil Contaminants, elaborado para o The Institute of $\mathrm{Pe}$ troleum, em 2002 (IP, 2002). Este relatório apresenta uma avaliação quantitativa de modelos de transporte de vapores do solo e, valida a sua performance com relação a medições esperadas para verificação da qualidade do ar ambiente. O guia inclui uma avaliação individual dos principais modelos disponíveis quanto a sua aplicabilidade, a calibração destes modelos utilizando dados específicos das áreas sob avaliação e recomendações quanto a utilização de modelos apropriados para a tomada de decisões.

Cenário Brasileiro: Com a ausência de uma regulamentação específica para a avaliação da intrusão de vapores, são seguidas as diretrizes para a gerenciamento de áreas contaminadas estabelecidas pela Resolução CONAMA 420/2009 (CONAMA, 2009). O CONAMA 420/2009 prevê a aplicação de modelos matemáticos (Ex.: PlaniIhas de cálculo da CETESB), para prever as concentrações de COVs em fase vapor, esperadas para o interior de edificações a partir de uma fonte de contaminação subsuperficial e assim determinar a necessidade de adoção de medidas de intervenção (CETESB, 2009). Entretanto, estudos demonstram que este método de análise pode ser conservador, visto que variáveis importantes, tais como biodegradação aeróbia, profundidade no nível d'água, fração de carbono orgânico no solo, porosidade do meio, dentre outras, não são incluídas ou são tratadas de uma maneira genérica (CHIARANDA, 2006; GOUVÊA 2011).

O Estado de São Paulo, único no Brasil a possuir uma diretriz própria para o gerenciamento de áreas contaminadas, regulamentada por meio da Decisão de Diretoria № 038/2017/C, de 07 de fevereiro de 2017 da CETESB, prevê, em alguns casos, a aquisição de dados de campo que incluem a amostragem de vapores: i) durante a implementação de programas de monitoramento preventivo, onde é possível incluir amostragens na zona não saturada ou de ar ambiente; ii) uso de normas internacionais para matrizes ambientais não previstas nas normas nacionais, como no caso, para amostras de vapores do solo; iii) em áreas contaminadas pela presença de COVs, o monitoramento poderá se dar a partir do ar da zona não saturada; iv) na 
etapa de Investigação Confirmatória, para áreas onde a Avaliação Preliminar identificar a possibilidade de uso de COVs, deverá ser previsto o mapeamento de COVs em fase vapor; v) adoção de vapores de comparação definidos na última atualização dos Regional Screening Levels - RSL desenvolvidos pela USEPA ou calculados por meio da Planilha de Avaliação de Riscos da CETESB (CETESB, 2017).

Existem também iniciativas privadas, como a NICOLE Brasil, organização não governamental liderada pelo setor industrial, que possui um grupo técnico de trabalho voltado ao desenvolvimento de estudos relacionados ao processo de intrusão de vapores. Em 2016, este grupo divulgou um White Paper, que foi elaborado com base nas melhores práticas adotadas nos Estados Unidos, adaptado às condições do meio físico verificadas no Brasil (NICOLE, 2016). Paralelamente, em 2016, a Associação Brasileira de Normas Técnicas (ABNT) formou um grupo técnico voltado ao desenvolvimento de normas específicas para a coleta ativa de amostras de gases e vapores do solo da zona vadosa, abaixo ou próximo de uma edificação.

\section{DESENVOLVIMENTO DE MODELOS CONCEITUAIS PARA AVALIAÇÃO DA INTRUSÃO DE VAPORES}

O desenvolvimento de modelos conceituais possui como objetivo principal a apresentação tridimensional de dados, de maneira mais abrangente e clara possível, incluindo todas as características relevantes, provendo a todas partes interessadas uma compreensão geral do cenário e uma avaliação quanto a potencial exposição aos compostos químicos de interesse presentes em fase vapor (NEW JERSEY, 2005). A seguir, serão abordados alguns aspectos específicos importantes que recentemente vem sendo incluídos de forma mais concisa nos modelos conceituais para explicar a presença ou ausência do processo de intrusão de vapores em áreas sob gerenciamento ambiental. 


\subsection{Avaliação de caminhos preferenciais}

No contexto da avaliação da intrusão de vapores subsuperficiais, caminhos preferenciais podem ser definidos como condutos de alta permeabilidade, que possuem a capacidade de transportar vapores a partir de uma área fonte para o interior das edificações (ITRC, 2007). Caminhos preferenciais podem ocorrer em locais onde existem redes de utilidades subterrâneas, que interceptem ou estejam sobrepostas a uma pluma de contaminação.

Instalações com características de alta permeabilidade, não necessariamente conectadas de forma direta a pluma de contaminação, também podem ter um impacto importante na migração e distribuição de COVs no interior de ambientes fechados. Os COVs podem migrar por meio de conduítes no interior de paredes, poços de elevadores, escadarias ou espaços abertos existentes em porões e sótãos. Esta migração pode resultar em uma distribuição anômala de COVs em cômodos ou pisos não diretamente adjacentes à área fonte. Adicionalmente, quando utilidades subterrâneas interceptam regiões onde existem plumas de contaminação, a água pode infiltrar-se nas tubulações, permitindo que a partição para a fase vapor ocorra no interior da rede. Nestes casos, os impactos podem ocorrer até mesmo em edificações lateralmente deslocadas em relação à pluma de contaminação.

Na maioria dos casos, a influência de caminhos preferenciais é identificada somente após a realização de investigações de detalhe que visam esclarecer situações incertas do modelo conceitual ou após medidas de mitigação falharem quanto ao controle dos riscos (GUO et al, 2015). Alguns guias sugerem que camadas de alta permeabilidade, de ocorrência natural (por exemplo, areia, cascalho ou rochas verticalmente fraturadas), também podem atuar como caminhos preferenciais para migração de vapores (API, 2005; ITRC, 2007; ITRC, 2014; USEPA, 2015).

\subsection{Presença de barreiras físicas}

Características inerentes ao meio físico, podem atuar como fator limitador para a intrusão de vapores. Exemplos de barreiras físicas incluem: lentes de água limpa na porção superior do aquífero, camadas de silte ou argila saturadas, posicionadas acima da fonte de COVs, processos de biodegradação e/ou outras transformações físicoquímicas que podem ocorrer na zona de vadosa, bases de edificações que possuam 
algum tipo de impermeabilização, ventilação ou indução de pressão positiva (MCHUGH et al., 2013). A Figura 03 a seguir ilustra exemplos de barreiras potenciais para a intrusão de vapores.

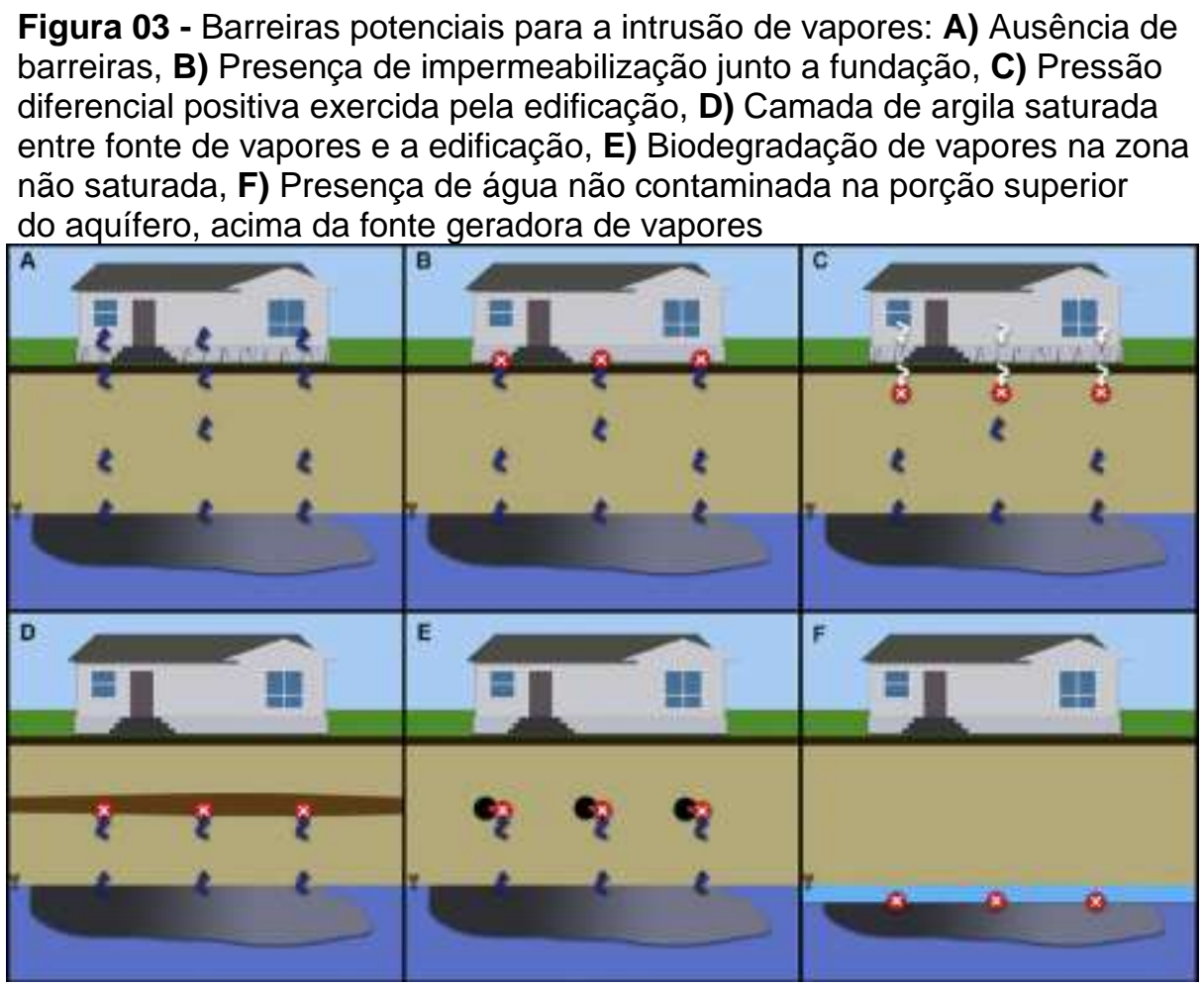

Fonte: Modificado de Mchugh et al. (2017)

A biodegradação aeróbica é bastante reconhecida e documentada como processo limitante para a migração de vapores de COVs de hidrocarbonetos de petróleo (API, 2008; CRCCARE, 2013; DAVIS, 2009; DEVAULL, 2007; HERS, 2002; ITRC, 2007; ITRC, 2014; USEPA, 2002; USEPA, 2015). Devaull, Salinitro e Gustafson (1997) sugerem que em condições onde as concentrações de oxigênio excedem 4\% e onde a concentração de nitrato dissolvido exceda 1 a 2 mg/L, o processo de biodegradação aeróbica pode ser mantido. Os resultados obtidos por Ziwck et al. (1997, apud HERS, 2002) sugerem que o processo de biodegradação aeróbica é significativamente diminuído à medida que o conteúdo de umidade presente no solo aumenta. Estes resultados são consistentes com os obtidos por Teixeira et al. (2009), que estudou o processo de oxidação aeróbica do metano na cobertura de três aterros sanitários no Brasil. Os resultados deste estudo sugerem uma relação inversa entre o grau de saturação no momento da coleta das amostras de solo analisadas e o número de bactérias metanotróficas presentes no perfil de solo. Estas evidências demonstram 
que a fase dissolvida de COVs está menos sujeita aos processos de biodegradação em condições aeróbicas e anaeróbicas na água subterrânea.

A biodegradação aeróbica de COVs utilizando aceptores de elétrons alternativos (ex. nitrato, sulfato) pode ocorrer na água subterrânea em taxas consideráveis, entretanto poucos estudos foram desenvolvidos para demonstrar a magnitude da biodegradação na zona não saturada utilizando estes aceptores (GOUVÊA, 2011). Outras linhas de evidência podem ser utilizadas para demonstrar a ocorrência de biodegradação na zona não saturada, tais como a presença de outros gases no solo. Durante o processo de biodegradação, o oxigênio será consumido e no seu lugar, será gerado dióxido de carbono (HERS, 2002).

A biodegradação aeróbica pode ser reconhecida pela assinatura ilustrada na Figura 04, onde as concentrações de COVs são altas próximas à fonte (fase dissolvida, livre ou residual), acompanhada pela redução de oxigênio e enriquecimento nas concentrações de dióxido de carbono. Acima da zona contaminada, as concentrações de dióxido de carbono e oxigênio retornam a condições esperadas para ambientes próximos a superfície. Dependendo da espessura da coluna de solo existente sobre a fonte de vapores, a biodegradação poderá anular completamente o processo de intrusão de vapores (DAVIS, 2009).

Figura 04 - Perfil característico de concentrações indicativo de biodegradação aeróbica

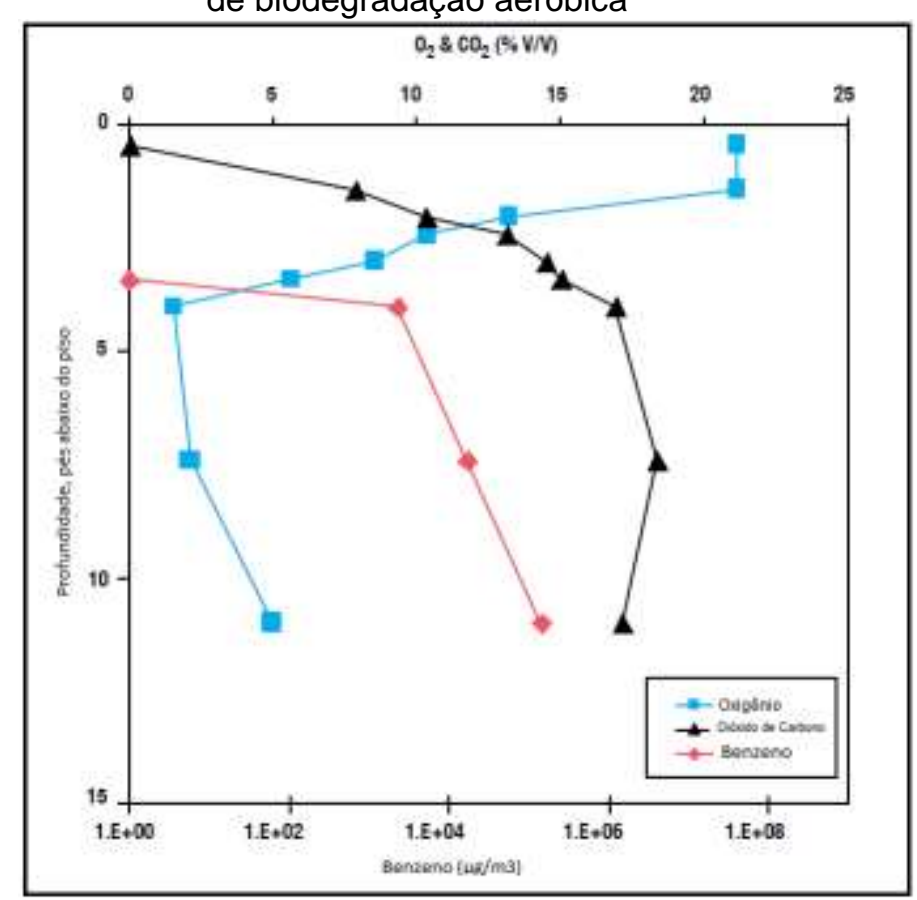

Fonte: Modificado de Davis (2009) 
Segundo alguns autores, a biodegradação aeróbica também pode ter um papel limitador quanto à migração de vapores de alguns COVs clorados, como por exemplo, cloreto de vinila (PATTERSON et al., 2013) e, em alguns locais, dependendo das condições presentes, cis-1,2-dicloroeteno (SCHMIDT et al., 2010).

\section{ABORDAGENS INOVADORAS PARA AVALIAÇÃO DA INTRUSÃO DE VAPORES}

A maior parte dos guias atuais recomenda uma abordagem baseada na adoção de múltiplas linhas de evidências, onde nenhum resultado é considerado definitivo (HEALTH CANADA, 2010, CRCCARE, 2013, IP 2002, ITRC 2007, ITRC 2014, USEPA 2002, USEPA 2015). Todos os dados disponíveis são avaliados para determinar possíveis impactos. Embora exista uma grande variedade de métodos, concentrações de COVs determinadas em matrizes ambientais são normalmente consideradas como principais linhas de evidência nos modelos conceituais. Linhas de evidência adicionais incluem fatores complexos, como a análise da variabilidade espacial e temporal das concentrações de COVs e a presença de fontes difusas não relacionadas às contaminações presentes no meio ambiente subterrâneo. Nos últimos anos, diversas abordagens inovadoras foram desenvolvidas para verificação destas situações, conforme os seguintes exemplos:

\subsection{Amostragens passivas com uso de materiais sorventes}

Comparado a técnicas que utilizam recipientes a vácuo (Ex. Summa Canisters ${ }^{\circledR}$, Bottle-Vacs $\left.{ }^{\circledR}\right)$, amostragens passivas que utilizam materiais sorventes possuem como vantagem o fácil manuseio, tamanho reduzido dos equipamentos e a possibilidade de adaptação a procedimentos de amostragem que requerem tempos de exposição superiores a 24 horas. Tempos de amostragens mais longos reduzem os efeitos da variabilidade temporal e os resultados obtidos representam uma concentração média dos COVs ao longo tempo.

Nos últimos anos, os estudos publicados se concentraram na validação de técnicas de amostragens passivas utilizando materiais sorventes (Ex. resinas, carvão ativado, Tenax ${ }^{\oplus}$, Carbotrap ${ }^{\circledast}$ para o monitoramento de COVs em períodos médios a longos de exposição, que podem variar desde semanas até meses (MCHUGH et al, 2017). Amostradores passivos tubulares, axiais e radiais (p.ex., tubos preenchidos 
com materiais absorventes específicos, como Beacon ${ }^{\circledR}$ e Radiello ${ }^{\circledR}$ vem sendo comumente utilizados para obter dados qualitativos e semi-quantitativos relacionados a distribuição e mapeamento das ocorrências de COVs nos vapores do solo (API, 2005; ITRC, 2007; ITRC, 2014; USEPA, 2002; USEPA, 2015).

\subsection{Coleta de amostras abaixo do piso (sub slab) com alto volume de purga}

Durante o mapeamento de vapores em uma edificação, espera-se que a distribuição das concentrações existentes abaixo do piso, apresente uma variação espacial maior do que a observada no ar ambiente, onde as concentrações tendem a ser homogêneas (EKLUND, 2010; MCALARY et al., 2010; MCHUGH et al., 2017; TURCZYNOWICZ et al, 2012). Como resultado, um número maior de pontos de amostragem é necessário para caracterizar a concentração média de COVs com um nível razoável de confiabilidade.

Para otimizar este processo, McAlary et al. (2010) desenvolveram um procedimento que prevê a realização de uma purga de alto volume a partir de um número reduzido de amostragens abaixo do piso (sub slab). Neste procedimento (Figura 05), uma bomba a vácuo é utilizada para realizar a purga de alto fluxo (por exemplo, 10 a $100 \mathrm{~L} / \mathrm{min})$. Como resultado, a amostra coletada irá fornecer uma média das concentrações de COVs presentes abaixo do piso, no entorno geral do ponto de amostragem. Adicionalmente, um instrumento de campo pode ser utilizado para medir as concentrações de COVs durante o fluxo de purga, fornecendo algumas informações importantes sobre a distribuição espacial de COVs abaixo do piso da edificação.

Figura 05 - Equipamento usado para amostragem abaixo do piso utilizando alto volume de purga e exemplo de dados de campo mostrando a presença de um "hot spot" a alguma distância da sonda

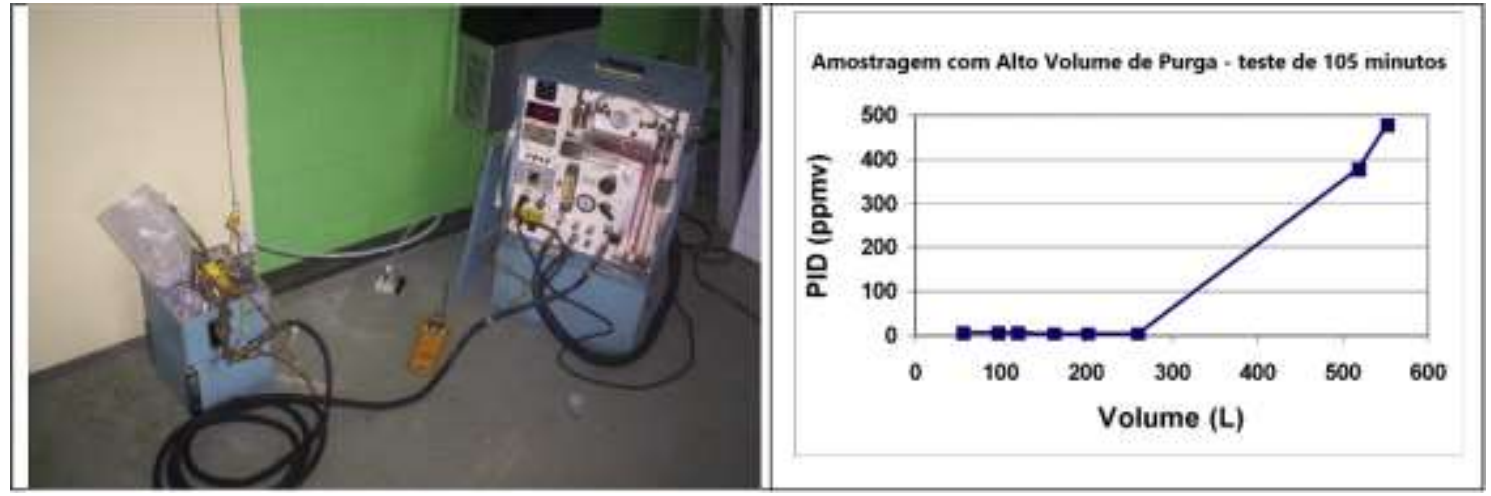

Fonte: Modificado de Mchugh et al. (2017) 


\subsection{Análises em tempo real}

A realização de análises em tempo real tem o potencial de otimizar o processo de investigação, permitindo que o pesquisador escolha de maneira mais assertiva a locação dos pontos de amostragens subsequentes, com base nos resultados das concentrações de contaminantes obtidas nas amostras anteriores. Esta rápida interação otimiza a capacidade de localizar a(s) fonte(s) de COVs detectados no ar interior relacionadas a intrusão de vapores. Este procedimento também pode ser utilizado para identificar a presença e avaliar taxas de emissões relacionadas a fontes internas (p. ex., materiais presentes no interior de residências, adesivos, produtos de limpeza, combustíveis, tintas, solventes), medindo assim a efetiva contribuição de cada tipo de fonte com relação às concentrações de COVs presentes no ar de ambientes fechados (BECKLEY et al., 2014; GORDER e DETTENMAIER, 2011; MCHUGH et al., 2017).

\subsection{Medição e controle de pressão exercida pela edificação}

Segundo McHugh et al. (2017), uma pequena diferença de pressão (< $5 \mathrm{~Pa}$ ) entre a edificação e a subsuperfície do terreno é suficiente para controlar a direção do fluxo de ar através das fundações. A aplicação de uma pequena pressão positiva é suficiente para anular o processo de intrusão de vapores, enquanto a existência de uma pequena pressão negativa é suficiente para ativar o processo. Assim, o monitoramento da pressão diferencial, pode fornecer informações importantes sobre as prováveis fontes dos COVs detectados.

Quando a pressão diferencial exercida é positiva, é provável que os COVs detectados no ar interior não estejam relacionados a contaminações presentes no meio subterrâneo, mas sim, associadas a presença de fontes internas ou ao background atmosférico. Na maioria das edificações, a pressão diferencial pode ser facilmente manipulada utilizando equipamentos simples, tais como ventiladores, sistemas de exaustão, aquecimento e de ar condicionado. Como resultado, a medição de concentrações de COVs no ar interior sob pressão diferencial pode proporcionar um melhor entendimento da dinâmica do processo de intrusão de vapores na área avaliada.

Caso existam fontes mistas, a mudança de fluxo entre as condições de pressão pode ser utilizada para estimar a contribuição de cada uma destas fontes de forma controlada (MCHUGH et al., 2017). Este método é previsto nos guias do ITRC (2014) 
e a USEPA (2015) como uma alternativa válida para distinguir concentrações de COVs relacionadas a intrusão de vapores, sejam por fontes internas ou por aquelas relacionadas a background.

\subsection{Utilização da análise piano para determinação de assinaturas químicas de hidrocarbonetos}

Análises convencionais em amostras de vapores do solo, como as baseadas na aplicação do Método EPA TO-15 (USEPA, 1999; NJDEP, 2017), possibilitam a determinação e a quantificação de um grupo relativamente limitado de hidrocarbonetos derivados de petróleo e de aditivos presentes na gasolina. Este tipo de análise não permite diferenciar concentrações associadas à presença de fontes subsuperficiais daquelas relacionadas a fontes existentes no interior das edificações (p.ex., produtos que contenham em sua composição compostos orgânicos voláteis ou semi-voláteis) ou ao background atmosférico local (UHLER; STOUT; MCCARTHY, 1999; WAIT, 2000).

Para possibilitar uma melhor compreensão quanto a natureza, origem e idade das concentrações detectadas em amostras de vapores de solo, foram desenvolvidos nas últimas duas décadas métodos específicos para a determinação de assinaturas químicas de hidrocarbonetos, que incluem a detecção de uma lista expandida de compostos alvo, possibilitando assim a realização de um refinamento do modelo conceitual esperado para o processo de intrusão de vapores nas áreas sob investigação (MORRISON; MURPHY; DOHERTY, 2006).

Neste contexto, a análise PIANO forense, baseada no refinamento do Método EPA TO-15 (USEPA, 1999; UHLER et al., 2003; DAVIS et. al., 2005; OUDJIK, 2005; PLANTZ et al., 2006; GALPERIN; KAPLAN, 2007; OUDIJK, 2009a; OUDIJK, 2009b; STOUT; DOUGLAS; UHLER, 2010; NJDEP, 2017), possibilita a identificação de até 1000 hidrocarbonetos voláteis diferentes, divididos em 5 classes de compostos principais (n-Parafinas, Iso-Parafinas, Aromáticos, Naftalenos e Olefinas). Compostos oxigenados normalmente encontrados na gasolina também são alvo do método (UHLER et al., 2003; DOUGLAS et al., 2007).

Para realização desta análise, utilizam-se técnicas de cromatografia gasosa purge-and-trap e espectrometria de massas (GS/MS) de forma combinada, operadas em modo de varredura completa e modo de monitoramento iônico, com limites de 
detecção inferiores às de análises convencionais. Durante o procedimento, são realizadas calibrações multivariadas para diagnosticar hidrocarbonetos geralmente ignorados em metodologias padrão (UHLER et al., 2003; PLANTZ et al., 2006).

Os resultados são apresentados em razões percentuais quanto ao peso, volume ou concentração molar, a partir do qual é obtido um perfil de distribuição entre os 5 grupos principais de constituintes de petróleo, que podem ser comparados a padrões pré-estabelecidos, possibilitando desta forma caracterizar e diferenciar tipos de gasolina, destilados de petróleo leves e produtos de alcatrão (NJDEP, 2017).

Os resultados de análises de múltiplas amostras são normalmente ilustrados em gráficos ou em diagramas do tipo estrela, onde a porcentagem relativa de cada grupo de compostos pode ser rapidamente comparada aos padrões existentes.

A Figura 06 ilustra um exemplo de comparação entre perfis cromatográficos referentes a padrões para gasolina e alcatrão, com perfis cromatográficos obtidos em amostras de vapores coletadas em uma área sob investigação. Nesta comparação é possível verificar que as amostras de vapores coletadas possuem uma assinatura química similar ao padrão de distribuição esperado para gasolina.

Figura 06 - Comparação entre perfis cromatográficos padrão e obtidos por meio de amostragens de vapores de solo

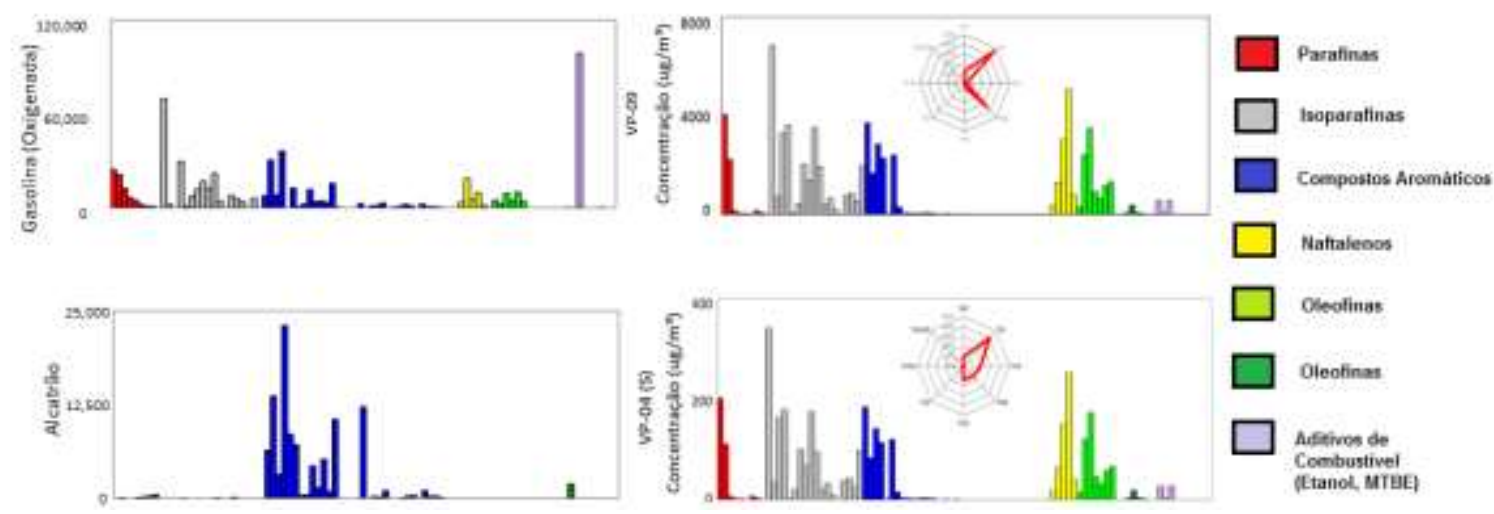

Fonte: o Autor

No contexto do processo de intrusão de vapores subsuperficiais, as assinaturas químicas determinadas nas análises podem ser utilizadas para distinguir diferentes fontes de concentrações de COV ou COSV (p.ex., fontes subsuperficiais, background atmosférico local e produtos químicos utilizados no interior das edificações) (MORRISON, 2000; MORRISON; MURPHY; DOHERTY, 2006). Adicionalmente, em função da susceptibilidade da degradação de um determinado grupo de compostos 
aos processos intempéricos ou de biodegradação, pode ser possível estimar a idade relativa da contaminação (MORRISON, 2000; MORRISON; MURPHY; DOHERTY, 2006; OUDIJK, 2009a; OUDIJK, 2009b).

A análise TO-15 convencional pode ser usada como uma ferramenta preliminar de avaliação e os dados podem ser usados para determinar a necessidade de realizar análises específicas, incluindo a determinação das assinaturas químicas dos hidrocarbonetos presentes. Normalmente, a análise PIANO forense pode ser realizada a partir dos mesmos procedimentos de coleta e amostragem empregados no método EPA TO-15 convencional (USEPA, 1999; NJDEP, 2017).

\subsection{Análise de isótopos estáveis}

A análise de isótopos em compostos específicos (ou CSIA - Compound-Specific Isotope Analysis) é uma abordagem relativamente recente para o cenário nacional, mas que vem sendo cada vez mais utilizada em várias aplicações ambientais (USEPA, 2008). O principal uso dos isótopos está relacionado à avaliação da degradação dos compostos químicos de interesse ambiental na água subterrânea. Com o sucesso obtido na aplicação dos CSIA em COVs dissolvidos em água subterrânea, surgiu maior interesse na utilização deste método para avaliar contaminações de COVs em fase vapor, quanto à determinação dos processos de atenuação na zona vadosa e o potencial para diferenciar as fontes presentes em ambientes fechados. Assim, o enriquecimento em isótopos gerados pela biodegradação, geralmente resulta em razões isotópicas distintas para a fonte subterrânea daquelas relacionadas a utilização de produtos de consumo (MCHUGH et al., 2011). A Figura 07 a seguir ilustra exemplos da aplicação desta tecnologia para distinção de fontes internas de fontes subsuperficiais para geração de vapores de Percloroetileno (PCE). 
Figura 07 - Exemplo do uso de CSIA para distinguir fontes internas e subsuperficiais de PCE

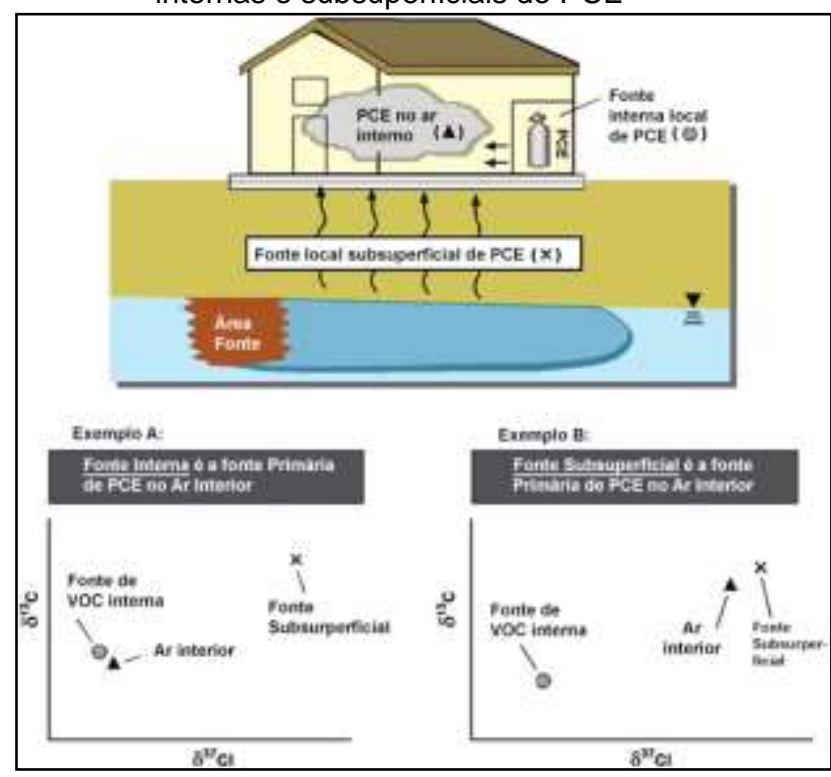

Fonte: Modificado de Mchugh (2011)

\subsection{Uso de gases traçadores}

O uso de traçadores é baseado na capacidade que estes gases possuem de "imitar" o comportamento dos vapores presentes na zona vadosa, em termos de dinâmica de fluxo subsuperficial, possibilitando assim um refinamento do modelo conceitual. Os gases traçadores podem ter origem natural ou antropogênica, podendo estar naturalmente presentes ou liberados em uma área controlada para realização deste tipo de avaliação. Exemplos de gases traçadores comumente utilizados nesta abordagem incluem o radônio, hexafluoreto de enxofre $\left(\mathrm{SF}_{6}\right)$ e o hélio. A utilização de gases traçadores é considerada uma técnica de aplicação simples e vem sendo amplamente utilizada para uma variedade de fins não necessariamente relacionados ao processo de intrusão de vapores, como por exemplo, a avaliação das taxas de troca do ar interno para verificação da eficiência energética em sistemas de controle de temperatura (ASTM 2001; EKLUND e SIMON, 2007; LOLL et al., 2016; LUNDEGARD et al., 2008; MCHUGH et al, 2008; MCHUGH et al., 2012; MCHUGH et al., 2017; LANGELAND et al., 2014; OLSON e CORSI, 2001; RIIS et al., 2010; SCHUVER e STECK, 2015). 


\subsection{Identificação de pontos de intrusão de vapores em edificações}

Em algumas edificações, a intrusão de vapores ocorre principalmente por meio de caminhos preferenciais. A identificação destes caminhos pode confirmar a ocorrência do processo e também proporcionar uma oportunidade para mitigação parcial do risco (por meio da vedação dos pontos de ingresso). Foram desenvolvidos vários métodos para identificar pontos de intrusão de vapores em edificações. Todos esses métodos requerem a indução de pressão diferencial negativa (em relação ao piso) para induzir fluxo advectivo através do ponto avaliado.

Técnicas mais comumente utilizadas para este tipo de abordagem incluem: i) avaliação a nível de ppb: Instrumentos de detecção de COVs, como o ppbRAE ${ }^{\circledR}$ ou similares, com capacidades de detecção de nível de ppb, podem ser utilizados para identificação de pontos de intrusão de vapores. Estes instrumentos fornecem uma medida semi-quantitativa em tempo real da concentração total de COVs no ar que está sendo monitorada; ii) termografia: Para aplicação deste método, utiliza-se um sensor para identificar diferenças de temperatura nas superfícies avaliadas. Quando as temperaturas do solo são inferiores às temperaturas internas, a intrusão de vapores pode resultar em temperaturas mais baixas nos pontos de intrusão. Entretanto, alguns autores apontam uma série de limitações quanto a aplicação deste método (MCHUGH et al, 2017; SEBASTIAN, 2009; LANGELAND et al, 2014); iii) hidrogênio: Este método consiste em uma liberação controlada de um gás traçador $\left(5 \% \mathrm{H}_{2} / 95 \% \mathrm{~N}_{2}\right)$ junto à potencial área fonte de vapores e a avaliação de pontos potenciais para intrusão de vapores com detector de hidrogênio. Este tipo equipamento é comumente utilizado para detectar vazamentos em gasodutos e outras utilidades. Destaca-se que este método requer pouco treinamento, utiliza equipamentos pouco onerosos e produz rápidos resultados (LOLL, 2008; LOLL, 2010; RIIS et al 2010; MCHUGH et al, 2017).

\subsection{Modelos disponíveis para avaliação da intrusão de vapores e cálculo de níveis alvo}

Existem basicamente três abordagens para avaliar o potencial risco relacionado ao processo de intrusão de vapores:

1) Coleta, analise e comparação dos valores obtidos em amostras de ar ambiente com os valores de referência estabelecidos; 
2) Compilação e análise de dados (amostragem de vapores no subsolo) e verificação entre as condições reais e as esperadas para a área;

3) Uso de modelos para prever concentrações esperadas para o ar do interior dos ambientes fechados, a partir da existência de fontes subsuperficiais de contaminação.

Estas abordagens não precisam ser mutuamente exclusivas e cada uma pode desempenhar um papel importante para a avaliação de potenciais impactos relacionados à intrusão de vapores (US EPA, 2002).

Visto que estabelecer uma rotina frequente para monitoramento da qualidade do ar no interior dos ambientes fechados pode ser tecnicamente e economicamente inviável, a utilização de modelos preditivos tem desempenhado um papel importante. As aplicações destes modelos incluem:

a) estimar o impacto da intrusão de vapores em áreas específicas;

b) identificar áreas que necessitam de análises mais específicas;

c) obter níveis alvo específicos para as áreas em estudo; e,

d) identificar como estes níveis poderão ser alterados em função de variações nas concentrações presentes em água subterrânea ou em função das características químicas dos compostos de interesse.

Apesar da limitação imposta pela USEPA com relação ao uso de modelos preditivos (USEPA 2002), as diretrizes divulgadas recentemente (USEPA 2015) procuram incentivar a utilização do maior número de linhas de evidências possíveis, as quais incluem o uso de modelos.

Segundo Picone (PICONE, 2012), os modelos preditivos podem ser divididos entre analíticos, que requerem a utilização de dados de entrada mais simples e, modelos numéricos, que exigem uma caracterização mais completa da área sob avaliação para determinação das variáveis específicas consideradas na rotina de cálculos.

A maioria dos modelos em uso são determinísticos. Eles dependem de parâmetros de referência para cada composto e tipo de solo avaliado. Alguns modelos analíticos também permitem uma abordagem probabilística, considerando a variabilidade e a incerteza relacionada aos parâmetros utilizados nos cálculos (PICONE, 2012).

Os modelos mais utilizados na prática são os modelos analíticos unidimensionais, que explicam a difusão e advecção de vapores e não consideram a biodegrada- 
ção (JOHNSON e ETTINGER, 1991; WAITZ et al., 1996). Todos os modelos disponíveis levam em consideração a difusão e a advecção da fase vapor, devido à existência de gradientes de pressão (JOHNSON e ETTINGER, 1991; JURY et al., 1984, JURY et al., 1990).

O modelo de J\&E e suas extensões (p. ex., API, 2005), são atualmente os modelos mundialmente mais utilizados para avaliar o processo de intrusão de vapores. O algoritmo de J\&E combina equações que determinam a partição dos contaminantes junto à fonte de vapores, transporte na zona vadosa, transporte através da laje de piso das edificações e a mistura com o ar presente no interior dos ambientes fechados. Os resultados irão depender dos dados utilizados para entrada, que consideram os dados específicos do solo local, características das edificações e parâmetros físicos e químicos dos compostos de interesse. Como resultado, o modelo fornece uma estimativa do fator de atenuação de vapores ( $\alpha$ ), que representa a razão entre a concentração medida diretamente no interior dos ambientes fechados e a concentração de vapores do solo em subsuperfície:

$$
\alpha=\frac{\left[\frac{D_{T}^{e f f} A_{B}}{Q_{B} L_{T}}\right] \exp \left(\frac{Q_{\text {soil }} L_{\text {crack }}}{D_{\text {crack }}^{e f f} \eta A_{B}}\right)}{\exp \left(\frac{Q_{\text {soil }} L_{\text {crack }}}{D_{\text {crack }}^{\text {eff }} \eta A_{B}}\right)+\left[\frac{D_{T}^{e f f} A_{B}}{Q_{B} L_{T}}\right]+\left[\frac{D_{T}^{e f f} A_{B}}{Q_{\text {soil }} L_{T}}\right]\left(\exp \left(\frac{Q_{\text {soil }} L_{\text {crack }}}{D_{\text {crack }}^{e f f} \eta A_{B}}\right)-1\right)}
$$

Onde $\alpha=\left(\mathrm{C}_{\text {indoor }} / \mathrm{C}_{\text {source }}\right)$, e:

$\mathbf{A}_{\mathbf{B}}=$ Área da superfície do ambiente fechado em contato com o solo $\left(\mathrm{m}^{2}\right)$;

$\mathbf{D}^{\text {eff }}{ }_{\text {crack }}=$ Coeficiente de difusão efetiva da fase vapor através de rachaduras das paredes e da fundação $\left[\mathrm{m}_{\text {crackeff }}{ }^{2} / \mathrm{d}\right]$;

$\mathbf{D}^{\text {eff }} \mathbf{T}=$ Coeficiente de difusão efetiva da fase vapor na coluna de solo presente entre a fundação e a fonte $\left[\mathrm{m}^{2} / \mathrm{d}\right]$;

$L_{\text {crack }}=$ Espessura da laje de piso do ambiente fechado $[\mathrm{m}]$;

$\mathbf{L}_{\mathbf{T}}=$ Distância (profundidade) até a fonte de vapores ou outro ponto de interesse abaixo da laje [m], medido a partir da laje do ambiente fechado em direção a fonte de vapores ou outro ponto de interesse;

$\mathbf{Q}_{\mathbf{B}}=$ Vazão volumétrica de $\operatorname{ar}\left[\mathrm{m}^{3} / \mathrm{d}\right]$. Geralmente estimado como o produto do volume do espaço fechado (VB $\left[\mathrm{m}^{3}\right]$ ) multiplicado pela razão de trocas do ar interno pelo ar externo (EB [1/d]);

$Q_{\text {soil }}=$ Fluxo de gás no subsolo dirigido por pressão para o ambiente fechado $\left[\mathrm{m}^{3} / \mathrm{d}\right]$; 
$\boldsymbol{\eta}$ = Fração da área superficial do espaço fechado aberta para intrusão de vapores $\left[\mathrm{m}^{2} / \mathrm{m}^{2}\right]$; comumente referida como "crack factor", pode ser estimada como a área total de trincas, fraturas, descontinuidades e perfurações existentes na superfície em contato com o solo, dividida pela área total em contato com o solo;

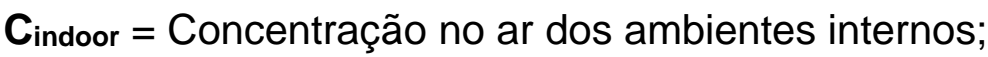

$\mathbf{C}_{\text {source }}=$ Concentração na Fonte

A Figura 08 ilustra a base conceitual do algoritmo e a relação entre os oito principais parâmetros de entrada no modelo primário.

Nota-se que a equação 1, representa a versão mais utilizada em termos práticos do algoritmo. Esta versão considera que a fonte de vapores está em estado estacionário, ou seja, as concentrações não variam ao longo do tempo. A equação que considera a redução das concentrações ao longo do tempo junto à área fonte de vapores também é apresentada no modelo publicado por J\&E.

Figura 08 - Base conceitual do algoritmo de Johnson e Ettinger e a relação entre os oito principais parâmetros de entrada no modelo primário

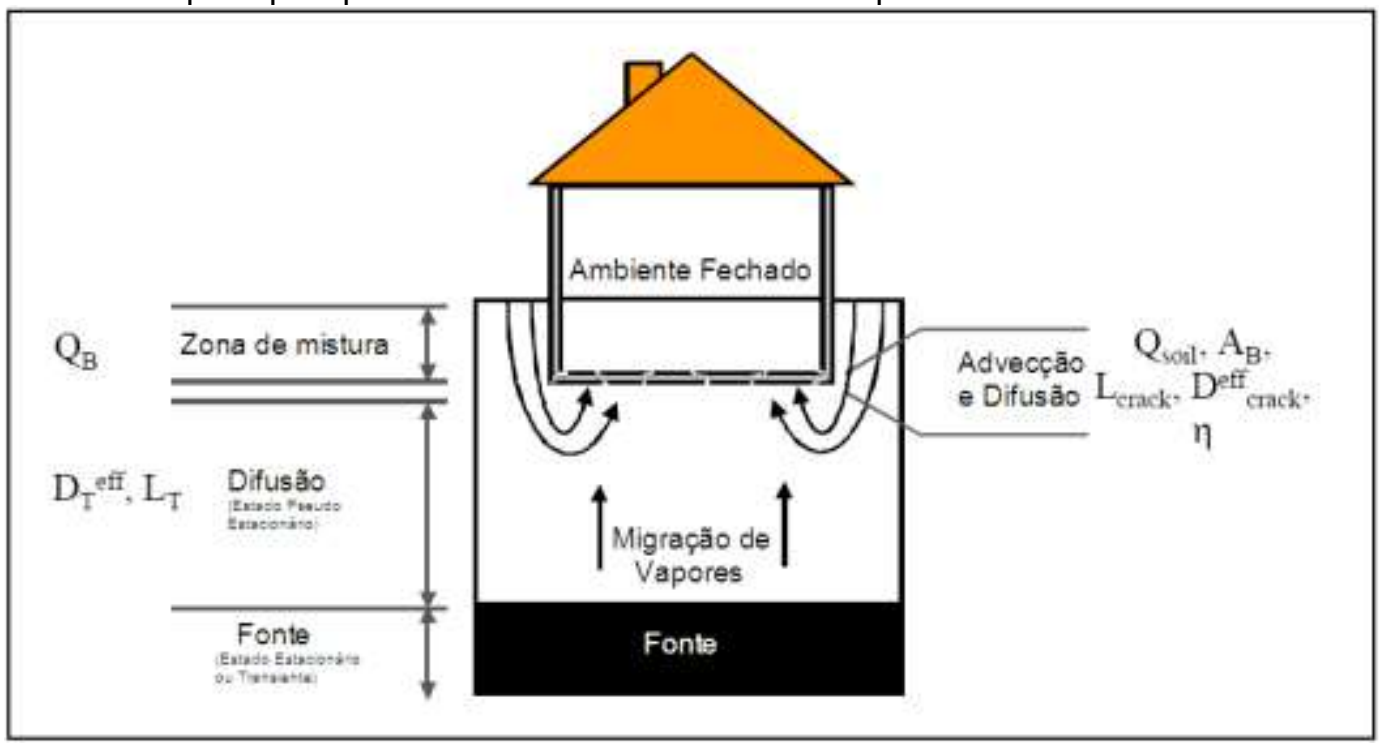

Fonte: Modificado de Johnson (2002)

O modelo VOLASOIL desenvolvido por WAITZ et al. (1996), atualizado por BAKKER et al. (2000), também considera diferenciais de pressão relacionados a gradientes de pressão atmosférica ou infiltração de água. Assim como o modelo Johnson e Ettinger, o VOLASOIL, assume a presença de um perfil homogêneo do solo. Ambos os modelos podem incluir a presença de fontes em fase livre (NAPL) como origem da 
fase de vapor (PICONE, 2012). Mais recentemente, a biodegradação aeróbica, incluindo o consumo de oxigênio, foi incorporada em modelos analíticos, como por exemplo, o BioVapor (DEVAULL, 2007; API, 2009).

O BioVapor (API, 2009), incorpora ao modelo de J\&E o potencial de biodegradação aeróbica na zona não saturada. Os resultados do modelo são calculados para intervalos da concentração de origem, as características dos solos na zona não saturada e os parâmetros físicos da construção avaliada. Em geral, os resultados deste modelo indicam que o potencial de intrusão de vapores em ambientes fechados é significativamente inferior em relação aos modelos que não consideram o processo de biodegradação (GOUVÊA, 2011).

Além dos modelos analíticos, foram desenvolvidos diversos modelos numéricos que levam em consideração a variação nas propriedades do solo ou em processos específicos (Tabela 01). Os modelos numéricos podem representar i) fatores físicos específicos, incluindo ventilação, barreiras, heterogeneidades ou a presença de fase livre não aquosa (DAVIS et al., 2005; FISCHER e UCHRIN, 1996; MENDOZA e FRIND, 1990a; MENDOZA e MCALARY, 1990; TILLMAN e WEAVER, 2007); ii) condições transitórias, tais como variações na pressão atmosférica ou no nível d'água (GARBESI e SEXTRO, 1989; MASSMANN e FARRIER, 1992); e iii) processos bioquímicos e fatores que os influenciam, incluindo a presença de camadas de turfa, solos anaeróbicos e barreiras que impeçam o fluxo de oxigênio (ABREU e JOHNSON, 2006; HERS et al., 2000; JOHNSON et al., 1999).

Modelos analíticos, como os introduzidos por JOHNSON e ETTINGER (1991) e WAITZ et al. (1996), fornecem ferramentas de avaliação para estimativas de risco, mas negligenciam propriedades físicas e processos significativos, como por exemplo a biodegradação. Por outro lado, modelos numéricos tridimensionais como os apresentados por Abreu e Johnson (2005) e Bozkurt et al. (2009) são por vezes muito complexos e exigentes em relação a dados de entrada específicos, que podem não estar disponíveis em situações reais de campo (PICONE, 2012).

\section{CONCLUSÕES}

A crescente preocupação com o processo de intrusão de vapores em áreas contaminadas pela presença de COVs no início dos anos 90, motivou o desenvolvimento de diretrizes e tecnologias especificas para avaliação desta via de exposição, 
principalmente em termos de verificação de riscos à saúde humana. Com isto, tornouse necessário o estabelecimento de quadros regulatórios para orientar as partes envolvidas no gerenciamento de áreas contaminadas quanto a processos de investigação e de tomada de decisão. Ao longo de três décadas de desenvolvimentos, países como Austrália, Canadá, Dinamarca, Estados Unidos e Reino Unido, estabeleceram quadros regulatórios específicos, envolvendo a obtenção de dados de campo, utilização de múltiplas linhas de evidência, interpretação de dados e recomendações quanto a adoção de medidas de mitigação para locais onde sejam identificadas situações potenciais de risco. Nestes locais, novas tecnologias e formas de abordagem vem sendo utilizadas de maneira bem-sucedida para elucidar os cenários de exposição, por meio do refinamento de modelos conceituais, auxiliando assim quanto a tomada de decisões. Em outros países, não se observa o estabelecimento de orientações específicas, seja para a aquisição de dados ou a para sua interpretação, sendo que a abordagem usual é focada em análise de risco a saúde humana e na utilização de modelagens. A situação no Brasil segue a mesma tendência. Foram identificados desenvolvimentos com relação ao quadro regulatório e tecnológico, porém existe um campo amplo de oportunidades para o estabelecimento de diretrizes e procedimentos específicos para avaliação do processo de intrusão de vapores. Espera-se que o desenvolvimento de regulamentações e abordagens específicas poderá auxiliar quanto a padronização do processo de gerenciamento ambiental de áreas contaminadas onde o processo de intrusão de vapores é relevante, tornando a percepção dos riscos mais realistas e assim possibilitar a seleção de técnicas mais eficientes, sustentáveis e economicamente viáveis para mitigação de riscos associados à intrusão de vapores.

\section{REFERÊNCIAS}

ABREU, L. D. V.; JOHNSON, P. C.. Effect of Vapor Source Building Separation and Building Construction on Soil Vapor Intrusion as Studied with a Tree-Dimensional Numerical Model. Environment Science \& Technology, Arizona State University, Tempe, v. 12, n. 39, p.4550-4561, 2005.

ABREU, L. D. V.; JOHNSON, P. C.. Simulating the Effect of Aerobic Biodegradation on Soil Vapor Intrusion into Buildings: Influence of Degradation Rate, Source Concentration, and Depth. Environment Science \& Technology, Arizona State University, Tempe, v. 40, n. 12, p.2304-2315, 2006. 
API - AMERICAN PETROLEUM INSTITUTE. A Pratical Strategy for Assessing the Subsurface Vapor-to-Indoor Air Migration Pathway at Petroleum Hydrocarbon Sites: Collecting and Interpreting Soil Gas Samples from Vadose Zone. Publication 4741. Washington D. C: Regulatory Analysis and Scientific Affairs, 2005. . Disponível em: < http://www.api.org/oil-and-natural-gas/environment/clean-water/ground-water/vapor-intrusion/vi-publication/assessing-vapor-intrusion >. Acesso em: 29 jan. 2018.

API - AMERICAN PETROLEUM INSTITUTE. BIOVAPOR-A 1-D Vapor Intrusion Model with Oxygen-Limited Aerobic Biodegradation Version 2.0. United States Of America: American Petroleum Institute, 2009. 58 p. Disponível em: http://www.api.org/oil-and-naturalgas/environment/clean-water/ground-water/vapor-intrusion/vi-publication/assessing-vaporintrusion. Acesso em: 29 jan. 2018.

ASTM - AMERICAN SOCIETY FOR TESTING AND MATERIALS - AMERICAN SOCIETY FOR TESTING AND MATERIALS. Standard Test Method for Determining Air Change in a Single Zone by Means of a Tracer Gas Dilution. Conshohocken, Pennsylvania: Annual Book of AMERICAN SOCIETY FOR TESTING AND MATERIALS Standards, 2001. Disponível em: < https://www.astm.org/Standards/E741.htm>. Acesso em: 29 jan. 2018.

BAKER, R.J., BAEHR, A.L. ; LAHVIS, M.A.. Estimation of hydrocarbon biodegradation rates in gasoline-contaminated sediment from measured respiration rates. Journal of Contaminant Hydrology, v. 41, p.175-192, 2000.

BECKLEY, L., GORDER, K., DETTENMAIER, E., RIVERA-DUARTE, I., MCHUGH, T.. Onsite gas chromatography/mass spectrometry (GC/MS) analysis to streamline vapor intrusion investigations. Environmental. Forensics, v. 15, p.234-243, 2014.

BOZKURT, O.; PENNEL, K.G; SUUBUERG, E.M..Simulation of the vapor intrusion process for nonhomogeneous soils using a three-dimensional numerical model. Groundwater Monitoring and Remediation, v. 29, p.92-104, 2009.

CRC FOR CONTAMINATION ASSESSMENT AND REMEDIATION OF THE ENVIRONMENT. Technical report 23: Petroleum hydrocarbon vapour intrusion assessment: Australian guidance. Salisbury: Environment Agency, 2013. 147 p. Disponível em: < http://www.crccare.com/files/dmfile/CRCCARETechreport23-PetroleumhydrocarbonvapourintrusionassessmentAustralianguidance2.pdf>. Acesso em: 29 jan. 2018.

HEALTH CANADA. Federal contaminated site risk assessment in Canada, part VII: Guidance for Soil Vapour Intrusion Assessment at Contaminated Sites. Ottawa: Health Canada, 2010. 152 p. Disponível em: <https://www.canada.ca/en/health-canada.html>. Acesso em: 29 jan. 2018.

CETESB - COMPANHIA DE TECNOLOGIA E SANEAMENTO AMBIENTAL DO ESTADO DE SÃO PAULO. Ações Corretivas Baseadas em Risco (ACBR) aplicadas a áreas contaminadas com hidrocarbonetos de petróleo e outros combustíveis líquidos. São Paulo, 2001. 80 p. Relatório Final.

CETESB - COMPANHIA DE TECNOLOGIA E SANEAMENTO AMBIENTAL DO ESTADO DE SÃO PAULO. Decisão de Diretoria № 263/2009. São Paulo, 2009. 55 p. Disponível em: $<$ http://www.cvs.saude.sp.gov.br/up/A\%C3\%A7\%C3\%B5es\%20corretiva\%20AC.pdf >. Acesso em: 29 jan. 2018.

CETESB - COMPANHIA DE TECNOLOGIA E SANEAMENTO AMBIENTAL DO ESTADO DE SÃO PAULO. Decisão de Diretoria № 38/2017/C. São Paulo, CETESB. 2017. 65 p. 
Disponível em: < http://www.cetesb.sp.gov.br/wp-content/uploads/2014/12/DD-038-2017-

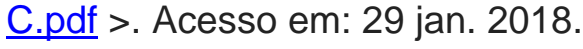

CHIARANDA, E. S. Volatilização dos compostos BTEX em ambientes subsuperficiais contaminados com gasolina e etanol: comparação entre concentrações medidas e simuladas. Florianópolis, 2006. 119 f. Dissertação (Mestrado) - Departamento de Engenharia Sanitária e Ambiental, Universidade Federal de Santa Catarina, Florianópolis, 2006.

CONAMA - CONSELHO NACIONAL DO MEIO AMBIENTE. Resolução № 420, de 28 de dezembro de 2009. Dispõe sobre critérios e valores orientadores de qualidade do solo quanto à presença de substâncias químicas e estabelece diretrizes para o gerenciamento ambiental de áreas contaminadas por essas substâncias em decorrência de atividades antrópicas. Brasil, 2009.

DANISH ENVIRONMENTAL PROTECTION AGENCY. 816: Feltafprøvning af sporgasmetode til brug for måling af transport af forureninger mellem renserier og tilstødende lejligheder. DANISH EPA, 2003. Disponível em: http://eng.mst.dk/. Acesso em: 29 jan. 2018.

DAVIS, A. et al. Use of Geochemical Forensics to Determine Release Eras of Petrochemicals to Groundwater. Environmental Forensics, New York, n. 6, p. 253-271, 2005.

DAVIS, G.B.; RAYNER, J.L.; TREFRY, M.G.; FISCHER, S.J; PATTERSON, B.M.. Measurement and modeling of temporal variations in hydrocarbon vapor behavior in a layered soil profile. Vadose Zone Journal, v. 4, p. 225-239, 2005.

DAVIS, R. V.. Update on Recent Studies and Proposed Screening Criteria for the Vapor-Intrusion Pathway. LUSTLine Bulletin, Massachusetts, n. 61, p.11-14, maio de 2009.

DEVAULL, G .E.; SALINITRO, J .P.; GUSTAFSON, J. B.. Benzene, toluene, ethylbenzene, and xylenes (BTEX) degradation in vadose zone soils during vapor transport: first order rate constants. In: CONFERENCE ON PETROLEUM HYDROCARBONS AND ORGANIC CHEMICALS IN GROUND WATER: PREVENTION, DETECTION, AND REMEDIATION., 1997, Houston, Texas. Proceedings... . Houston, Texas: NWWA/API, 1997.

DEVAULL, G. E.. Indoor Vapor Intrusion with Oxygen-Limited Biodegradation for a Subsurface Gasoline Source. Environmental Science \& Technology, v. 41, n. 9, p.3241-3248, 2007.

DOUGLAS, G. D. et al. Chemical fingerprinting methods. In: MURPHY, B.; MORRISON, R.. Introduction to Environmental Forensics. 2. ed. New York: Academic Press, 2007. p. 312454.

EKLUND, B., SIMON, M., Concentration of tetrachloroethylene in indoor air at a former dry cleaner facility as a function of subsurface contamination e a case study. Journal of Air Waste Management, v 57, 753-760, 2007.

EKLUND, B. Typical spatial and temporal variability in vapor intrusion data sets and implications for regulatory policy. In: INTERNATIONAL CONFERENCE ON REMEDIATION OF CHLORINATED AND RECALCITRANT COMPOUNDS, 7., 2010, Monterey. Proceedings... Monterey: Batelle, 2010.

FISCHER, D; UCHRIN, C.G.. Laboratory simulation of VOC entry in to residence basements from soil gas. Environment Science \& Technology, v. 30 p.2598-2603, 1996. 
GALPERIN, Y.; KAPLAN, I. R.. Forensics Environmental Geochemistry in Dispute Resolution: Case History 1: Age Dating a Gasoline Plume at a Service Station in Geneva. Environmental Forensics, New York, p.339-349, 2007.

GARBESI, K.; SEXTRO, R.G..Modeling and field evidence of pressure-driven entry of soil gas into a house through permeable below-grade walls. ES and T Contents, v. 23, 14811487, 1989.

GORDER, K.A., DETTENMAIER, E.M.. Portable GC/MS methods to evaluate sources of cVOC contamination in indoor air. Ground Water Monitoring, v. 31, 113-119, 2011.

GOUVÊA, J. C. R. G. Jr. 2011. Influência dos parâmetros do meio físico sobre as concentrações de referência para intrusão de vapores de BTEX em ambientes fechados. 2011. 135 f. Dissertação (Mestrado) - Tecnologias Ambientais, Instituto de Pesquisas Tecnológicas do Estado de São Paulo, 2011.

GUO, Y., HOLTON, C., LUO, H., DAHLEN, P., GORDER, K., DETTENMAIER, E., JOHNSON, P. Identification of alternative vapor intrusion pathways using controlled pressure testing, soil gas monitoring, and screening model calculations. .Environmental Science \& Technology, v. 49, n. 22, p. 13472-13482, 2015.

HERS, I; ATWATER, J; LI, L; ZAPF-GILJE, R.. Evaluation of vadose zone biodegradation of BTX vapours. Journal of Contaminant Hydrology, v. 46 p. 233- 264, 2000.

HERS, E. D. et al. TECHNICAL REPORT: Vapor Transfer of Soil Contaminants: The Institute of Petroleum, 2002. 118 p. P5-18/TR.

ITRC - THE INTERSTATE TECHNOLOGY \& REGULATORY COUNCIL. Vapor Intrusion Pathway: a practical guideline. Washington D.C: The Interstate Technology \& Regulatory Council, 2007. 172 p. Disponível em: https://www.itrcweb.org/documents/VI-1.pdf. Acesso em: 29 jan. 2018.

ITRC - THE INTERSTATE TECHNOLOGY \& REGULATORY COUNCIL. Petroleum Vapor Intrusion: Fundamentals of Screening, Investigation and Management. Washington D.C: The Interstate Technology \& Regulatory Council, 2014. 391 p. Disponível em: https://www.itrcweb.org/PetroleumVI-Guidance/. Acesso em: 29 jan. 2018.

JOHNSON, P. C.; ETTINGER, R. A.. Heuristic Model for Predicting the Intrusion Rate of Contaminant Vapors into Buildings. Environmental Science \& Technology, Houston, v. 25, n. 8, p.1445-1452, 1991.

JOHNSON, P.C.; KEMBLOWSKI, M.W; JOHNSON, R.L.. Assessing the significance of subsurface contaminant vapor migration to enclosed spaces: site-specific alternatives to generic estimates. Soil and Sediment Contamination, v 8 p. 389-421, 1999.

JURY, W.A.; SPENCER, W.F.; FARMER, W.J.. Behavior assessment model for trace organics in soil: II. Chemical classification and parameter sensitivity. Journal of Environmental Quality, v. 13, p. 567-572, 1984.

JURY, W.A.; RUSSO, D.; STREILE, G.; EL ABD, H.. Evaluation of volatilization by organic chemicals residing below the soil surface. Water Resources Research, v. 26, p. 13-20, 1990.

LANGELAND, M., HYLDEGAARD, W., KREILGAARD, S . Metoder til sporing af indtrængningsveje for poreluft til indeklima. Danish EPA, 2014, 46 p. 
LOLL, P.; LARSEN, C.; GRØN, M.. Opløsningsmidlers vej til indeklimaet. Vand \& Jord, v. 3, p. 93-96, 2008.

LOLL, P; LARSEN, P; LARSEN, C. Tracking vapor intrusion pathways e an active tracer gas test. In: INTERNATIONAL CONFERENCE ON REMEDIATION OF CHLORINATED AND RECALCITRANT COMPOUNDS, 7., 2010, Monterey. Proceedings... . Monterey: Batelle, 2010.

LOLL, P. et al. Tracer estimation of attenuation factors at vapor intrusion sites. In: INTERNATIONAL CONFERENCE ON REMEDIATION OF CHLORINATED AND RECALCITRANT COMPOUNDS, 10., 2016, Palm Springs. Proceedings... . Palm Springs: Batelle, 2016.

LUNDEGARD, P. D.; JOHNSON, P. C.; DAHLEN, P.. Oxygen Transport From the Atmosphere to Soil Gas Beneath a Slab-on-Grade Foundation Overlying Petroleum-Impacted Soil. Environmental Science \& Technology, Fullerton, v. 42, n. 15, p.5534-5540, 2008.

MASSMANN, J.; FARRIER, D.F.. Effects of atmospheric pressure on gas transport in the vadose zone. Water Resources Research, v. 28, p. 777-791, 1992.

MCALARY, T.; NICHOLSON, P. J.; LEE, Y. K., BERTRAND, D. D.; TRUPP, G.. High purge volume sampling e a new paradigm for subslab soil gas monitoring. Groundwater Monitoring, v. 32, n. 2, 73-85, 2010.

MCHUGH, T.; NICKELS, TIM., N.. FINAL REPORT: Detailed Field Investigation of Vapor. Houston: GSI Environmental, Inc., 2008. 338 p. ESTCP Project ER-0423.

MCHUGH, T.; KUDER, T.; FIORENZA, S.; GORDER, K.; DETTENMAIER, E.; PHILP, P.. Application of CSIA to Distinguish Between Vapor Intrusion and Indoor Sources of VOCs. Environmental Science Technology, v. 45, p. 5952-5958, 2011.

MCHUGH, T.E.; BECKLEY, L.; BAILEY, D.; GORDER, K.; DETTENMAIER, E.; RIVERADUARTE, I.; BROCK, S.; MACGREGOR, I.C.. Evaluation of vapor intrusion using controlled building pressure. Environment Science \& Technology, v. 46, p. 4792-4799, 2012.

MCHUGH, T.; BECKLEY, L.; BAILEY, D. Influence of shallow geology on volatile organic chemical attenuation from groundwater to deep soil gas. Groundwater Monitoring, v. 45, p. 92-100, 2013.

MCHUGH, T.; LOLL, P ; EKLUND, B. Recent advances in vapor intrusion site investigations. Journal of Environmental Management, 1-10, 2017.

MENDOZA, C.A.; FRIND, E.O.. Advective-dispersive transport of dense organic vapors in the unsaturated zone. 1. Model development. Water Resources Research, v. 26 p. 379387, 1990a.

MENDOZA, C.A.; MCALARY, T.A.. Modeling of ground-water contamination caused by organic solvent vapors, Ground Water, v. 28, p. 199-206, 1990.

MORRISON, Robert D.. Critical Review of Environmental Forensics: Part II. Environmental Forensics, New York, v. 1, n. 4, p.175-195, dez. 2000.

MORRISON, R. D.; MURPHY, B. I.; DOHERTY, R. E.. Environmental Forensics: contaminant specific guide. New York: Elsevier, 2006. 
NEW JERSEY. Diane Groth. NJDEP Vapor Intrusion Committee (Org.). Vapor intrusion guidance. New Jersey, 2005. 283 p. Disponível em: http://www.nj.gov/dep/srp/guidance/vaporintrusion/. Acesso em: 29 jan. 2018.

NEW JERSEY DEPARTMENT OF ENVIRONMENTAL PROTECTION. Technical guidance document: Commingled Plume Technical Guidance Document. 1 ed. New Jersey, 2017. 105 p. Disponível em: http://www.nj.gov/dep/srp/guidance/srra/commingled plume guidance.pdf. Acesso em: 13 mar. 2018.

NICOLE BRASIL - Network For Industrially Contaminated Land in Latin America. José C. R. Gouvêa. Jr. Intrusão de vapores em ambientes fechados: conceitos básicos, avaliação e gerenciamento de áreas contaminadas no Brasil. Grupo Técnico de Intrusão de Vapores. São Paulo, 2016. 88p.

OLSON, D.A.; CORSI R.L.. Characterizing exposure to chemicals from soil vapor intrusion using a two-compartment model. Atmospheric. Environment, v. 35, p. 4201-4209, 2001.

OUDIJK, G. Fingerprinting and Age-Dating of Gasoline Release: A Case Study. Environmental Forensics, New York, v. 6, n. 1, p. 91-99, 2005.

OUDIJK, G. Age Dating Heating-Oil Releases: Part 1. Heating Oil Composition and Subsurface Weathering. Environmental Forensics, New York, v. 1, n. 10, p.107-119, 2009 a.

OUDIJK, G. Age Dating Heating-Oil Releases: Part 2. Assessing Weathering and Release Time Frames through Chemistry, Geology, and Site History. Environmental Forensics, New York, v. 10, n. 1, p.120-131, 2009b.

PATTERSON, B.M., ARAVENA, R., DAVIS, G.B., FURNESS, A.J., BASTOW, T.P., BOUCHARD, D.. Multiple lines of evidence to demonstrate vinyl chloride aerobic biodegradation in the vadose zone, and factors controlling rates. Journal of Contaminant. Hydrology, v. 153, p. 69-77, 2013.

PICONE, Sara. Transport and biodegradation of volatile organic compounds: influence on vapor intrusion into buildings. 2012. 163 f. Tese (Doutorado) - Curso Socio-economic and Natural Sciences Of The Environment, The Netherlands Research School For The Socioeconomic And Natural Sciences Of The Environment, Wageningen University, Wageningen, 2012.

PLANTZ, Gina M. et al. Analytical techniques for vapor intrusion pathway assessments: An environmental forensic approach. In: A\&WMA SPECIALTY CONFERENCE: VAPOR

INTRUSION: THE NEXT GREAT ENVIRONMENTAL CHALLENGE-AN UPDATE, 1., 2006, Los Angeles. Proceedings... . Los Angeles, 2006.

RIIS, C. et al. Vapor intrusion through sewer systems: migration pathways of chlorinated solvents from groundwater to indoor air, G-021, in remediation of chlorinated and recalcitrant Compounds. In: INTERNATIONAL CONFERENCE ON REMEDIATION OF CHLORINATED AND RECALCITRANT COMPOUNDS, 7., 2010, Monterey. Proceedings... . Monterey: Batelle 2010.

SCHMIDT, K., AUGENSTEIN, T., HEIDINGER, M., ERTI, S., TIEHM, A.. Aerobic biodegradation of cis-1,2-dichloroethene as sole carbon source: stable carbon isotope fractionation and growth characteristics. Chemosphere, v 78 p. 527-532, 2010. 
SCHUVER, H.J., STECK, D.J.. Cost-effective rapid and long-term screening of chemical vapor intrusion $(\mathrm{CVI})$ potential: across both space and time. Remediation Journal, v 25, p. 27 53, 2015.

SEBASTIAN, Walter. Kontrol af indeklimasikring ved termografi med undertryk: Et metodestudie. In: ATV JORD OG GRUNDVAND - TEMADAG 9. MARTS 2009 - PORELUFT OG

INDEKLIMA, 1., 2009, Copenhague. NIRÁS. Copenhague: Bygge Og Miljøteknik A/s, 2009. p. 1 - 22. Disponível em: http://www.atv-jord-grundvand.dk/. Acesso em: 12 mar. 2018.

STOUT, S. A.; DOUGLAS, G. S.; UHLER, A. D.. Assessing Temporal and Spatial Variations of Gasoline-Impacted Groundwater Using Relative Mole Fractions and PIANO Fingerprinting. Environmental Forensics, New York, v. 1, n. 11, p.328-341, 2010.

TEIXEIRA, C. E. et al. Estudos sobre a Oxidação Aeróbia do Metano na Cobertura de Três Aterros Sanitários no Brasil. Engenharia Sanitária e Ambiental, Universidade de Caxias do Sul, Caxias do Sul, v. 14, n. 1, p.99-108, 2009.

THE INSTITUTE OF PETROLEUM. P5-018/TR: Vapour Transfer of Soil Contaminants. 2002. 118 p. Disponível em: http://webarchive.nationalarchives.gov.uk/20100408233916/http://publications.environment-agency.gov.uk/pdf/SP5-018TR-e-p.pdf. Acesso em: 29 jan. 2018.

TILLMAN, J.F.D; WEAVER, J.W.. Temporal moisture content variability beneath and external to a building and the potential effects on vapor intrusion risk assessment. Science of the Total Environment, v. 379 p. 1-15, 2007.

TURCZYNOWICZ, L., PISANIELLO, D., WILLIAMSON, T.. Health risk assessment and vapor intrusion: a review and Australian perspective. Human and Ecological Risk Assessment, v.18, p. 984-1013, 2012.

UHLER, A. D.; STOUT, S. A.; MCCARTHY, K. J.. Increase success of assessments at petroleum sites in 5 steps. Soil \& Groundwater Cleanup, v. 1, n. 1, p.13-19, 1999.

UHLER, R. M. et al. Molecular fingerprinting of gasoline by a modified EPA 8260 gas chromatography/mass spectrometry method. International Journal Of Environmental Analytical Chemistry. Ottawa, p. 1-20. 2003.

USEPA - UNITED STATES ENVIRONMENTAL PROTECTION AGENCY. EPA 600/625/R96/010B: Compendium of Methods for the Determination of Toxic Organic Compounds in Ambient Air: Method TO-15. 2 ed. Cincinnati, 1999. 67 p. Disponível em:

https://www3.epa.gov/ttnamti1/files/ambient/airtox/to-15r.pdf. Acesso em: 13 mar. 2018.

USEPA - UNITED STATES ENVIRONMENTAL PROTECTION AGENCY . A Guide for Assessing Biodegradation and Source Identification of Organic Groundwater Contaminants Using Compound Specific Isotope Analysis (CSIA).. United States Of America: United States Environmental Protection Agency, 2008, 140 P. Disponível em: https://nepis.epa.gov . Acesso em: 29 jan. 2018.

USEPA - UNITED STATES ENVIRONMENTAL PROTECTION AGENCY . Draft Guidance for Evaluating the Vapor Intrusion to Indoor Air Pathway from Groundwater and Soils. United States Of America: United States Environmental Protection Agency, 2002. 53 p. Disponível em: www. https://archive.epa.gov/epawaste/hazard/web/pdf/appa-c.pdf. Acesso em: 29 jan. 2018. 
USEPA - UNITED STATES ENVIRONMENTAL PROTECTION AGENCY . Technical Guide for Addressing Petroleum Vapor Intrusion at Leaking Underground Storage Tank

Sites. United States Of America: United States Environmental Protection Agency, 2015. 129 p. Disponível em: https://www.epa.gov/ust/technical-guide-addressing-petroleum-vapor-intrusion-leaking-underground-storage-tank-sites. Acesso em: 29 jan. 2018.

WAIT, D. A.. Evolution of organic analytical methods in environmental forensic chemistry. Environmental Forensics, New York, v. 1, n. 1, p. 37-46, 2000.

WAITZ, M.;FREIJER, J.I.; KREULE, P.;SWARTJES, F.A..The VOLASOIL risk assessment model based on CSOIL for soils contaminated with volatile compounds. Netherlands: National Institute for Public Health and The Environment, 1996. 